Artigo Original

\title{
CARACTERÍSTICAS ANDROLÓGICAS E SEMINAIS DE CÃES DA RAÇA PASTOR BELGA MALINOIS
}

\author{
Autor: Christian Garcia Amantino', Nívea de Mattos Góes Vieira², Silvia Ferrari²,A.
}

1Aluno de Iniciação Científica da Faculdade de Medicina Veterinária da Universidade Anhembi Morumbi. 2Médica Veterinária, doutoras em reprodução animal pela Faculdade de Medicina Veterinária da USP.

\section{Informações do artigo}

Palavras Chave:

Exame andrológico;

Pastor Belga Malinois;

Parâmetros seminais.

\section{Resumo}

A realização do exame andrológico em cães, prévio à sua seleção e/ou utilização em um esquema de reprodução natural ou artificial é de grande importância, e é composto pela anamnese, exame clínico geral, exame do sistema reprodutivo, exame da libido, exame do sêmen e exames complementares, se necessário. No presente estudo, foram avaliados 11 cães adultos da raça Pastor Belga Malinois, com a finalidade de padronizar as características seminais e andrológicas da raça em questão, já que há grande variação das características entre as múltiplas raças de cães de diferentes portes, e escassa literatura sobre raças específicas. Como resultado, a avaliação dos testículos revelou o tamanho médio do testículo direito de $2,7 \pm 0,44 \mathrm{~cm}$ de largura, $2,73 \pm 0,40 \mathrm{~cm}$ de espessura e $4,44 \pm 0,44 \mathrm{~cm}$ de comprimento, e do esquerdo de $2,60 \pm 0,26 \mathrm{~cm}$ de largura, $2,84 \pm 0,34 \mathrm{~cm}$ de espessura e $4,48 \pm 0,51 \mathrm{~cm}$ de comprimento. O sêmen dos animais apresentou uma média de $88 \%$ de movimento retilíneo e progressivo, 4,6 de vigor e 9,55\% de espermatozoides anormais. Todavia, em relação ao volume do ejaculado e à concentração espermática houve uma grande variação entre os animais, 5 cães apresentaram valores de concentração abaixo de 100x106 sptz/ml, enquanto os demais se situaram acima deste valor. Conclui-se que a concentração espermática e o volume total do sêmen apresentaram variação, devido à variação do volume da secreção prostática, que foi maior principalmente em animais com mais de 5 anos com Hiperplasia Prostática Benigna, os demais parâmetros espermáticos estão de acordo com os padrões da espécie canina.



Silvia Ferrari - e-mail: silviaferrari2000@hotmail.com - https://orcid.org/0000-0002-9917-2843

DOI: https://doi.org/10.31415/bjns.v2i3.70 - Artigo recebido em: 18 de junho de 2019; aceito em 09 de Julho de 2019; publicado em 30 de setembro de 2019. Brazilian Journal of Natural Sciences, Vol. 2, N.3, setembro 2019. Disponível online a partir de 30 de setembro de 2019, ISSN 2595-0584. www.bjns.com.br. Todos os autores contribuíram igualmente com o artigo. Os autores declaram não haver conflito de interesse. Este é um artigo de acesso aberto sob a licença CC - BY: http://creativecommons.org/licenses/by/4.0 


\section{Article ID}

Keywords:

Andrological

examination; Belgian

Shepherd Malinois;

Seminal parameters.

\begin{abstract}
Performing the andrological examination in dogs prior to selection and / or use in a natural or artificial reproduction scheme is of great importance, and consists of anamnesis, general clinical examination, reproductive system examination, libido examination, semen and further examinations if necessary. In the present study, 11 adult dogs of the Belgian Shepherd Malinois breed were evaluated in order to standardize the seminal and andrological characteristics of the breed in question, since there is great variation of characteristics between the multiple breeds of dogs of different sizes, and scarce literature. about specific races. As a result, the evaluation of the test is revealed the average right testis size of $2.7 \pm 0.44 \mathrm{~cm}$ in width, $2.73 \pm 0.40 \mathrm{~cm}$ in thickness and $4.44 \pm 0.44 \mathrm{~cm}$ in length, and the left of $2,60 \pm 0,26 \mathrm{~cm}$ wide, $2,84 \pm 0,34 \mathrm{~cm}$ thick and $4,48 \pm 0,51 \mathrm{~cm}$ long. The semen of the animals presented an average of $88 \%$ of rectilinear and progressive movement, 4,6 of vigor and 9,55\% of abnormal sperm. However, regarding the ejaculate volume and sperm concentration there was a great variation between the animals, 5 dogs presented concentration values below $100 \times 106 \mathrm{sptz} / \mathrm{ml}$, while the others were above this value. It was concluded that the sperm concentration and the total semen volume presented variation, due to the variation of the volume of the prostatic secretion, which was higher mainly in animals over 5 years with Benign Prostatic Hyperplasia, the other sperm parameters are in agreement with the Canine species patterns.
\end{abstract}

\section{Introdução}

A avaliação da aptidão reprodutiva de um macho destinado à reprodução fundamenta-se na observação da saúde geral, saúde genética e saúde do sistema genital [1]. Segundo Cunha [2], a realização do exame andrológico em cães, prévio à sua seleção e/ou utilização em um esquema de reprodução artificial é de grande importância, sendo ainda indicada em outras circunstâncias como: compra ou venda de reprodutores, seleção de doadores para uso em programas de inseminação artificial ou diagnóstico de patologias do sistema genital masculino. Neste sentido Ferrari; Romano [3] diagnosticaram um tumor de células de Leydig em testículo de cão de 2,5 anos de idade durante o exame andrológico, retirando o animal da reprodução.

A análise reprodutiva do macho canino é composta de uma anamnese detalhada que inclua avaliação do comportamento reprodutivo, exame físico, avaliação do sêmen, dosagens hormonais, ultrassonografia testicular e prostática, e citologia aspirativa por agulha fina [4]. A análise do sêmen deve ser incluída na avaliação andrológica para detectar possíveis problemas de infertilidade ou subfertilidade, além de fazer parte da rotina pré- cobertura ou inseminação em cães [2]. A determinação das características seminais é extremamente importante na avaliação clínica reprodutiva de cães, visando aumentar o sucesso dos programas de acasalamento pela utilização de animais com qualidades espermáticas satisfatórias [5].

Existe um padrão das características do sêmen de cães, relatado por Mies Filho [6], que observa a grande variação do volume do sêmen de cão (2 a $40 \mathrm{ml}$ ), fato também relatado no manual para exame andrológico do CBRA (1), no qual o volume total do sêmen de cães varia de $1,5 \mathrm{a} 80 \mathrm{ml}$. Ainda este manual descreve a cor do sêmen como branca, acinzentada, odor 'suis generis', motilidade espermática igual ou superior a $70 \%$, vigor igual ou superior a 3 , concentração com grande variação, de 20 a $300 \times 106$ espermatozoides $/ \mathrm{ml}$, e espermatozoides morfologicamente normais 
iguais ou superiores a 70\%. Porém, a espécie canina apresenta grande variação entre raças e portes dos animais, que impacta na variação das características do sêmen, principalmente do volume do ejaculado.

Netto [7], avaliou o ejaculado de 24 cães da raça Boxer, e desprezando a $1^{\circ}$ e $3^{\circ}$ frações do sêmen, encontrou a média de volume da $2^{\circ}$ fração rica em espermatozoides igual a 2,06 $\pm 0,66 \mathrm{ml}$. A média da motilidade espermática foi de 76,28 \pm $5,97 \%$. Em relação à concentração espermática, encontrou 484,18 x $106 \pm 96,76$ e 19,15 $\pm 5,28 \%$ de patologia espermática.

Também Silva [8], avaliando a $2^{\circ}$ fração do ejaculado de 6 cães da raça Fila Brasileiro, encontrou 2,2 $\pm 1,3 \mathrm{ml}$ de volume, 589,7 $\pm 248,7$ milhões de espermatozoides por $\mathrm{ml}$ de concentração, 85,8 $\pm 3,7 \%$ de motilidade espermática, 5 de vigor e 85,6 $\pm 4,5 \%$ de espermatozoides morfologicamente normais.

Silva et al. [9] relatam em seu estudo a escassez de trabalhos de andrologia específicos da raça Golden Retriever, para comparar com o resultado da avaliação andrológica de 13 animais da raça.

Em face da grande diversidade das raças caninas, e da escassez de estudos andrológicos específicos das diversas raças, faz-se necessária a padronização das características seminais de cada raça, a fim de otimizar a escolha dos reprodutores.

Por conseguinte, o objetivo desse estudo é realizar o exame andrológico dos cães da raça Pastor Belga Malinois, a fim de relatar as particularidades seminais da raça, além de descrever características específicas do aparelho reprodutivo.

\section{Material e Método}

Foram avaliados 11 cães da raça Pastor Belga Malinois, com a faixa etária dentro de 2 a 8 anos que pertencem ao Canil da Polícia do Exército, localizado no Município de Osasco e Barueri, Estado de São Paulo. Todos os cães são alimentados única e exclusivamente com a ração Royal Canin ${ }^{\circ}$ Maxi Adult, e são treinados para diversas funções, dentre elas faro, proteção e guarda.

Além disso, realizou-se o exame clínico (anamnese e exame físico geral e específico) onde se avaliou estado geral, mucosas (oral e ocular), nível de hidratação através do turgor cutâneo de gradil costal, auscultação pulmonar e cardíaca, palpação de linfonodos (submandibulares, pré escapulares e poplíteos) e temperatura corpórea. $\mathrm{Na}$ realização do exame físico específico do aparelho reprodutor foi ressaltada palpação e inspeção de pênis, prepúcio, testículo, epidídmo, bolsa escrotal e próstata; medição de comprimento, largura e espessura de testículos; além da análise do sêmen propriamente dita que foi dividida em Análise Macroscópica e Análise Microscópica.

As coletas foram realizadas no período da tarde, em dias quentes e secos. Cada cão forneceu 1 ejaculado, tendo-se a certeza que não foi realizada nenhuma coleta ou cobertura ao menos 72 horas antes. O sêmen foi colhido por meio da manipulação do pênis com a mão enluvada. Não foi realizada a separação das 3 frações, a amostra foi depositada em um funil plástico acoplado à mamadeira contendo tubo coletor falcon imerso em água a $37^{\circ} \mathrm{C}$. Imediatamente após a coleta, o tubo foi levado ao banho maria, e o exame macroscópico do sêmen (volume, cor, aspecto, odor, $\mathrm{pH}$ ) foi realizado, onde foi avaliado o volume através da verificação da graduação do tubo coletor como descrito por Cunha (2), a avaliação de cor e aspecto foi feita de forma subjetiva através da simples observação da amostra, foi utilizado a olfação para avaliar-se o odor. $\mathrm{O}$ pH foi mensurado através da fita de $\mathrm{pH}$. Logo após a análise macroscópica foi realizado o exame microscópico do sêmen (motilidade retilínea e progressiva, vigor, concentração e avaliação da morfóloga espermática).

Em relação ao exame microscópico do sêmen, a motilidade retilínea e progressiva e vigor foram avaliados de forma subjetiva, colocando-se uma gota de sêmen sobre lâmina aquecida, sendo esta recoberta por lamínula. $\mathrm{O}$ exame foi realizado sob microscopia. O resultado obtido deve ser expresso em porcentagem (0-100) para a motilidade e um escore de zero a cinco (0-5) para vigor [2].

Além da motilidade retilínea e progressiva e vigor, foi realizada a avaliação da concentração e morfologia espermática. A avaliação da concentração espermática foi realizada através da contagem do número de espermatozoides da fração mais concentrada, depositada no fundo do tubo 
falcon, utilizando-se a câmara de "Neubauer", antes o sêmen foi diluído em solução de formol salino na proporção padrão para a espécie canina (1:20). Já a avaliação morfológica foi realizada através da técnica de câmara úmida, foi feita a diluição de 3 gotas de sêmen em $1 \mathrm{ml}$ de solução de PBS acrescido de formol a 10\%, colocando uma gota entre lâmina e lamínula, vedando as bordas com esmalte de unha, e avaliando 200 células 24 horas depois.

Para verificar uma possível padronização das características reprodutivas da raça, foi feita a média e desvio-padrão de todas as características avaliadas dos animais examinados.

\section{Resultados}

Através da anamnese foi possível identificar 2 animais cruzaram e tiveram filhos, o número de filhotes por ninhada do cão 6 foi de 3 ninhadas, com uma média de 5 filhotes saudáveis por ninhada, e o cão 4 com apenas 1 ninhada, com 11 filhotes hígidos.
$\mathrm{Na}$ realização do exame físico geral e específico do aparelho reprodutor de modo geral não houve alterações em auscultação cardíaca e respiratória, a temperatura corpórea variou entre $37,8^{\circ} \mathrm{C}$ a $39,7^{\circ} \mathrm{C}$ levando-se em consideração que a temperatura ambiente nos dias da coleta estava alta (média de $30^{\circ} \mathrm{C}$ ) e clima seco, todos os linfonodos avaliados não estavam reativos, turgor cutâneo normal indicando nível de desidratação adequado e estado geral ótimo em todos os cães.

Durante o exame físico específico do aparelho reprodutor não foi identificado nada digno de nota em relação a palpação e inspeção de pênis, prepúcio, testículo, e bolsa escrotal em nenhum animal, porém foi notado o aumento da cabeça do epidídimo esquerdo do cão 10 e aumento de próstata de alguns cães, verificado pela palpação digital retal (tabela $\mathbf{1}$ ).

Tabela 1: Valores de largura (L), espessura (E) e comprimento $(\mathrm{C})$ dos testículos em centímetros e descrição do tamanho da próstata em cada animal, de acordo com a idade dos cães do canil da Polícia do Exército.

\begin{tabular}{|c|c|c|c|c|c|c|c|c|}
\hline \multirow[b]{2}{*}{ Animal } & \multirow[b]{2}{*}{ Idade } & \multirow[b]{2}{*}{ Próstata } & \multicolumn{3}{|c|}{ Testículo Direito } & \multicolumn{3}{|c|}{ Testículo Esquerdo } \\
\hline & & & $\mathrm{L}$ & $\mathrm{E}$ & $\mathrm{C}$ & $\mathrm{L}$ & $\mathrm{E}$ & $\mathrm{C}$ \\
\hline 1 & $2 \mathrm{a}$ & n.d.n.1 & 2,3 & 3,0 & 4,2 & 2,4 & 2,7 & 4,0 \\
\hline 2 & 2 a $6 \mathrm{~m}$ & n.d.n. & 2,4 & 2,3 & 3,9 & 2,5 & 2,5 & 4,0 \\
\hline 3 & 2 a $10 \mathrm{~m}$ & aument.2 & 2,5 & 1,8 & 4,4 & 2,2 & 2,4 & 4,2 \\
\hline 4 & $3 a$ & n.d.n. & 3,0 & 2,9 & 4,8 & 2,9 & 2,7 & 4,1 \\
\hline 5 & $4 \mathrm{a} 1 \mathrm{~m}$ & n.d.n & 2,6 & 2,7 & 5,0 & 2,5 & 3,1 & 5,0 \\
\hline 6 & $5 \mathrm{a}$ & aumentada & 2,8 & 3,0 & 5,3 & 2,9 & 3,1 & 5,3 \\
\hline 7 & $7 \mathrm{a}$ & aumentada & 2,5 & 2,7 & 4,5 & 2,3 & 2,4 & 4,3 \\
\hline 8 & 7 a $2 \mathrm{~m}$ & n.d.n & 2,2 & 2,8 & 4,3 & 2,6 & 3,5 & 4,2 \\
\hline 9 & $8 \mathrm{a}$ & aumentada & 2,9 & 2,6 & 4,5 & 3,0 & 3,0 & 4,5 \\
\hline 10 & $8 \mathrm{a}$ & aumentada & 2,7 & 3,0 & 4,0 & 2,6 & 3,0 & 4,3 \\
\hline 11 & 8 a $9 \mathrm{~m}$ & aumentada & 3,8 & 3,3 & 4,0 & 2,8 & 2,9 & 5,4 \\
\hline \multicolumn{3}{|c|}{ Média } & 2,7000 & 2,7364 & 4,4455 & 2,6091 & 2,8455 & 4,4818 \\
\hline \multicolumn{3}{|c|}{ Desvio-padrão } & 0,4405 & 0,4056 & 0,4413 & 0,2625 & 0,3417 & 0,5115 \\
\hline
\end{tabular}

Tabela 1. ${ }^{1}$ n.d.n. nada digno de nota. ${ }^{2}$ Aumentada. ${ }^{3}$ Cabeça de epidídimo esquerdo aumentada

Nas tabelas 2 e 3 estão detalhados os resultados das avaliações microscópica e macroscópica do sêmen dos cães da Polícia do Exército. 
Tabela 2.

\begin{tabular}{|c|c|c|c|c|}
\hline \multirow{2}{*}{ Animal } & M.R.P. & Vigor & Concentração (Em milhões) & Morfologia \\
\cline { 4 - 5 } & & & & (D.T) \\
\hline 1 & $70 \%$ & 4 & $3,25 \mathrm{sptz} / \mathrm{ml}$ & $15,5 \%$ \\
\hline 2 & $90 \%$ & 5 & $462,00 \mathrm{sptz} / \mathrm{ml}$ & $3,0 \%$ \\
\hline 3 & $90 \%$ & 5 & $111,25 \mathrm{sptz} / \mathrm{ml}$ & $7,0 \%$ \\
\hline 4 & $90 \%$ & 5 & $28,50 \mathrm{sptz} / \mathrm{ml}$ & $13,0 \%$ \\
\hline 5 & $90 \%$ & 5 & $73,75 \mathrm{sptz} / \mathrm{ml}$ & $6,0 \%$ \\
\hline 6 & $90 \%$ & 5 & $187,50 \mathrm{sptz} / \mathrm{ml}$ & $15,5 \%$ \\
\hline 7 & $90 \%$ & 5 & $242,50 \mathrm{sptz} / \mathrm{ml}$ & $6,5 \%$ \\
\hline 8 & $90 \%$ & 5 & $27,50 \mathrm{sptz} / \mathrm{ml}$ & $15,0 \%$ \\
\hline 9 & $90 \%$ & 5 & $232,50 \mathrm{sptz} / \mathrm{ml}$ & $7,5 \%$ \\
\hline 10 & $90 \%$ & 3 & $68,75 \mathrm{sptz} / \mathrm{ml}$ & $6,5 \%$ \\
\hline 11 & $90 \%$ & 4 & $102,50 \mathrm{sptz} / \mathrm{ml}$ & $6,5 \%$ \\
\hline Média & $88 \%$ & 4,6364 & 139,9773 & $9,55 \%$ \\
\hline Desvio-padrão & 0,0603 & 0,6742 & 134,3590 & 0,0468 \\
\hline
\end{tabular}

Tabela 2. Avaliação microscópica do sêmen dos cães do canil da Polícia do Exército

Tabela 3.

\begin{tabular}{|c|c|c|c|c|c|c|}
\hline \multirow{2}{*}{ Animal } & \multicolumn{2}{|c|}{ Volume } & \multirow{2}{*}{ Cor } & \multirow{2}{*}{ Odor } & \multirow{2}{*}{ Aspecto } & \multirow{2}{*}{$\mathrm{pH}$} \\
\hline & Fr. Rica & Fr. Total & & & & \\
\hline 1 & $1 \mathrm{ml}$ & $1 \mathrm{ml}$ & Esbranquiçado & ${ }^{\star} S . G$ & Soroso & 6,5 \\
\hline 2 & $2 \mathrm{ml}$ & $2 \mathrm{ml}$ & Esbranquiçado & ${ }^{\star} S . G$ & Soroso & 6,5 \\
\hline 3 & $6 \mathrm{ml}$ & $7 \mathrm{ml}$ & Esbranquiçado & ${ }^{*} S . G$ & Soroso & 6,5 \\
\hline 4 & $3 \mathrm{ml}$ & $7 \mathrm{ml}$ & Esbranquiçado & ${ }^{\star}$ S.G & Soroso & 6,5 \\
\hline 5 & $6 \mathrm{ml}$ & $6 \mathrm{ml}$ & Esbranquiçado & ${ }^{\star} S . G$ & Soroso & 6,0 \\
\hline 6 & $3 \mathrm{ml}$ & $20 \mathrm{ml}$ & Esbranquiçado & ${ }^{*}$ S.G & Soroso & 6,5 \\
\hline 7 & $3 \mathrm{ml}$ & $20 \mathrm{ml}$ & Esbranquiçado & ${ }^{*} S . G$ & Soroso & 7,0 \\
\hline 8 & $3 \mathrm{ml}$ & $20 \mathrm{ml}$ & Esbranquiçado & ${ }^{\star} S . G$ & Soroso & 6,5 \\
\hline 9 & $6 \mathrm{ml}$ & $16 \mathrm{ml}$ & Esbranquiçado & ${ }^{*} S . G$ & Soroso & 6,5 \\
\hline 10 & $2 \mathrm{ml}$ & $20 \mathrm{ml}$ & Esbranquiçado & ${ }^{*} S . G$ & Soroso & 7,0 \\
\hline 11 & $4,5 \mathrm{ml}$ & $25 \mathrm{ml}$ & Avermelhado & ${ }^{\star} S . G$ & Soroso & 6,5 \\
\hline Média & 3,5909 & 13,0909 & & & & 6,5455 \\
\hline Desvio Padrão & 1,77226101 & 8,5726839 & & & & 0,2697 \\
\hline
\end{tabular}

Tabela 3: Avaliação macroscópica do sêmen dos cães do canil da Polícia do Exército.

${ }^{\star}$ S.G (Suis Generis)

\section{Discussão}

A avaliação dos testículos revelou o tamanho médio do testículo direito de $2,7 \pm 0,44 \mathrm{~cm}$ de lar- gura, $2,73 \pm 0,40 \mathrm{~cm}$ de espessura e $4,44 \pm 0,44 \mathrm{~cm}$ de comprimento, e do esquerdo de $2,60 \pm 0,26$ $\mathrm{cm}$ de largura, $2,84 \pm 0,34 \mathrm{~cm}$ de espessura e $4,48 \pm 0,51 \mathrm{~cm}$ de comprimento. Como o tamanho 
dos testículos é variável devido às diferenças raciais dos cães, espera-se com este estudo fornecer parâmetros do testículo para a raça Pastor Belga Malinois, e concorda-se com Silva et al. [9] que relatam em seu estudo a escassez de trabalhos de andrologia específicos da raça para comparação dos resultados obtidos.

De acordo com a palpação retal da próstata, notou-se o seu aumento nos cães acima de 5 anos de idade, apesar do cão 3 também demonstrar essa característica, tendo apenas 2 anos e 10 meses de idade. Este fato está de acordo com estudo elaborado por Johnston et. al [10], que afirma que $16 \%$ dos cães aos 2 anos de idade apresentaram hiperplasia prostática benigna - HPB (tabela 1).

Trabalhos demonstram que $80 \%$ dos cães com mais de cinco anos de idade são portadores de hiperplasia prostática benigna [11]. No presente estudo os cães demonstram indícios de aumento de próstata a partir de 5 anos de idade, vale ressaltar que a avaliação da próstata foi feita através de palpação digtal retal, e para o diagnóstico definitivo o ideal seria a realização do exame ultrassonográfico e histológico. Segundo Krakowski e colaboradores [12], a HPB não altera a qualidade do sêmen em cães.

Em relação ao Movimento Retilíneo e Progressivo (tabela 2) os animais apresentaram uma média de $88 \%$, quanto ao Vigor o valor médio foi de 4,6 e no que se refere a morfologia espermática, os cães demonstraram uma média de 9,55\% de espermatozoides anormais. Estes valores estão de acordo com SOUZA [13], porém estes dados se referem a cães da raça Pasto Alemão, e também dentro dos padrões estabelecidos pelo CBRA [1], para a espécie canina. Ainda segundo Johnston et al.[10], a motilidade retilínea progressiva normal do cão é acima de 70\%, e a taxa de espermatozoides anormais deve ser igual ou menor que $20 \%$.

Todavia, em relação à concentração espermática houve uma grande disparidade entre os animais, os cães 1, 4, 5, 8 e 10 mostraram valores abaixo de $100 \times 10^{6} \mathrm{sptz} / \mathrm{ml}$, enquanto os demais se situaram acima deste valor, com destaque para os cães 2 e 7 , com $462 \times 10^{6} \mathrm{sptz} / \mathrm{ml}$ e $242,50 \times 10^{6}$ $\mathrm{sptz} / \mathrm{ml}$, respectivamente. Souza e colaboradores (13) também obtiveram a concentração dos espermatozoides de sêmen de cães da raça Pastor Alemão variando de $18,2 \times 10^{6} \mathrm{sptz} / \mathrm{ml}$ a $326,8 \times$
$10^{6} \mathrm{sptz} / \mathrm{ml}$. Segundo Johnston et al. (10), a concentração do sêmen do cão depende da quantidade de fluido prostático coletado, e varia de 4 a 400 milhões de espermatozoides por mililitros. No presente estudo a fração rica do ejaculado não foi colhida separadamente das demais frações, apenas observou-se e utilizou-se o volume de sêmen mais concentrado do final do tubo falcon para a avaliação da concentração, por isso houve a grande variação de concentração espermática dentre os animais utilizados.

Verificou-se no presente estudo um aumento do volume total do ejaculado em relação à idade do animal. Sabe-se que o pico da função secretória da próstata acontece aos 4 anos de idade [10] e no presente estudo os animais com 5 anos ou mais apresentaram grande volume total do ejaculado, que pode estar relacionado com aumento de próstata que apresentaram à palpação retal. Iquer-Ouada e Verstegen [14] administraram finasteride para tratamento da HPB em cães e observaram após o tratamento uma diminuição do volume da secreção prostática e aumento da concentração de espermatozoides nos animais tratados, associados à diminuição do volume prostático.

\section{Conclusão}

Conclui-se com este trabalho que as características macro e microscópicas do sêmen dos cães da raça Pastor Belga Malinoir estão dentro dos padrões da espécie canina. A concentração espermática e o volume total do sêmen apresentaram variação, devido à variação do volume da secreção prostática, que foi maior principalmente em animais com mais de 5 anos. O tamanho dos testículos dos cães foi descrito no presente trabalho, fornecendo um padrão para o exame andrológico da raça.

\section{Agradecimentos}

Agradecemos à Universidade Anhembi Morumbi pelo fomento deste estudo através do Programa Institucional de Bolsa de Iniciação Científica Anhembi Morumbi (PIBIC/AM). Agradecemos também ao $2^{\circ}$ Sargento Adriano da Silva Souza do $22^{\circ}$ Depósito de Suprimento, ao Capitão Felipe Soares Borges e Capi- 
tão Otávio Augusto Brioschi Soares do $2^{\circ}$ Batalhão da Polícia do Exército pela disponibilidade de tempo e espaço para a realização das coletas, além de disponibilizar e auxiliar no manejo e contenção dos animais para a realização do estudo.

\section{Referências}

1. COLÉGIO Brasileiro de reprodução Animal- Manual para exame andrológico e avaliação de sêmen animal. 3 ed. Belo Horizonte: CBRA, 2013.

2. CUNHA, I.C.N Exame Andrológico do cão. Jornal Brasileiro de Ciência Animal JBCA, 2008; 1:49-65.

3. FERRARI, S.; ROMANO, M.A. Tumor de células de leydig em cão: relato de caso. A hora veterinária,2008; 27(161):17-9.

4. DOMINGOS, T.C.S; SALOMÃO, M.C. Meios de diagnóstico das principais afecções testiculares em cães: revisão de literatura. Rev. Bras. Reprod. Anim., Belo Horizonte,2011; 35(4): 393-9.

5. GABRIEL, A.M.A.; JESUS, V.L.T.; ROSA A.C.; FOLHADELA, D.S.; FOLHADELA, I. M. Características físicas e morfológicas do sêmen canino. Revista Brasileira de Reprodução Animal,1999; 23(3): 239-40.

6. MIES FILHO, A. Inseminação Artificial, ed. Sulina, Porto Alegre, vol.2, 1987.

7. NETTO, M.C.L. Avaliação das características seminais de cães da raça boxer submetidos a dietas suplementadas com vitamina $\mathrm{E}$ e levedo de cerveja. Dissertação de mestrado. São Paulo, 2001.

8. SILVA, A.S.F. Caracterização genética, andrológica e congelabilidade do sêmen em cães da raça fila brasileiro. Tese de doutorado, Belo Horizonte, 2012.

9. SILVA, B.L.M.; COSTA, B.K.; SILVA, M.E.A.; COSTA, M.E.; PADILHA, F.N.; LOPES, F.G. Características seminais de cães da raça Golden Retriever, criados no município de Londrina, PR. $42^{\circ}$ Congresso Bras. de Medicina Veterinária e $1^{\circ}$ Congresso Sul-Brasileiro da ANCLIVEPA 31/10 a 02/11 de 2015 - Curitiba - PR

10. JOHNSTON, S.D.; KUSTRITS, M.V.R.; OLSON, P.N.S. Chapter 16. Semen collection, evaluation and preservation. IN: Canine and feline theriogenology. Filadelfia: W.B. Saunders, 2001.

11. JOHNSTON, S.D.; KAMOLPATANA, K.; ROOT-KUSTRITS, M.V., JOHNSTON, G. R. Prostatic disorders in the dog. Animal Reproduction Science, 2000; 60-61: 405-15.

12. KRAKOWSKI, L.; WACHOCKA, A., BRODZKI, P.; WRONA, Z.; PIECH, T.; WAWRON, W.; CHALABIS-MAZUREKA A. Sperm quality and selected biochemical parameters of seminal fluid in dogs with benign prostatic hyperplasia. Animal Reproduction Science, 2015 ; 160:120-5. doi: 10.1016/j.anireprosci.2015.07.014

13. SOUZA, J.A.D.; Spicciati,W.; Visintin,J.A.; Barnabe,V.H.; Barnabe, R.C.; Características seminais de cães da raça Pastor Alemão. Brasilian Journal of Veterinary Research and Animal Science, 1995: 32(3): 181-6.

14.IQUER-OUADA, M.; VERSTEGEN, J. P. Effect of finasteride (Proscar MD) on seminal composition, prostate function and fertility in male dogs. Journal of Reproduction and Fertility Suppl , 1997; 51: 139-49. 